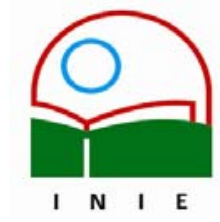

\title{
UNA TRAYECTORIA DE CALIDAD Y APORTE A LA EDUCACIÓN:
}

HISTORIA DEL INIE

\section{Por Marta Rojas Porras}

Hoy, el Instituto nos ha convocado a la celebración de sus 25 años. Una edad de juventud y madurez. Un espacio oportuno y obligado para recordar su gestación, su nacimiento y algunos aspectos de su trayectoria y desarrollo: un recorrido por lo más sobresaliente de su historia.

A fines de los años setenta, un grupo de profesores de la Facultad de Educación, liderados por el Dr. Carlos German Paniagua y el magister Fernando Castro, lucharon por la creación de un instituto donde se impulsara la investigación educativa. Para este propósito, se busca el apoyo del Ministerio de Educación Pública. Tanto la Licda. María Eugenia Dengo y el Lic. Marvin Herrera, Ministra y Viceministro de Educación, respectivamente, confían en que el proyecto cumplirá las expectativas educativas, no sólo para la Universidad, sino también para el país, y lo acogen con entusiasmo.

La promesa de la creación de este Instituto favorecía especialmente dos frentes:

En el ámbito universitario, se estimularía al docente de la Facultad de Educación para que se convirtiera en un investigador de su quehacer, con lo cual sus nuevas experiencias estarían respaldadas por informaciones y resultados probados científicamente. Además, se 
generaría la investigación interdisciplinaria en ciencias de la educación, se fortalecerían los programas de formación docente y administrativa de la Facultad y aquellos destinados a la acción social de la Universidad de Costa Rica.

En cuanto a la trascendencia nacional, el Instituto sería una valiosa fuente de asesoramiento, en el campo educativo, para el Ministerio de Educación Pública, que se nutriría de los resultados de estudios para la toma de decisiones.

Con estos antecedentes, el Consejo Universitario aprueba el Reglamento del Centro para el Mejoramiento de la Educación Costarricense (CIMEC) el 24 de setiembre de 1979. Al año siguiente, en febrero de 1980, éste, ya como Instituto de Investigación para el Mejoramiento de la Educación Costarricense (IIMEC), abre oficialmente sus puertas en una modesta oficina de la Facultad de Educación.

El Dr. Juan Manuel Esquivel Alfaro, designado como su primer Director, consciente de la realidad, enfrenta las dificultades materiales y, desde sus primeros pasos, le imprime a la Institución un sello de prestigio, seriedad y liderazgo en la investigación educativa costarricense.

En honor a la verdad, también las carencias se suplen gracias a la dedicación y trabajo en equipo de quienes acompañaron al Dr. Esquivel en esta empresa académica y filial: Zayra Méndez, Marta Picado, Alicia Gurdián, Jesús Ugalde, Sonia Alpízar y Marisol Nieto -todos profesores de la Facultad de Educación-, Liliana Quesada, Vilma Delgado, Natalia Campos, Damaris Fajardo, Teresita Peralta, Ana María González y Marta Rojas -investigadoras invitadas, destacadas por el Ministerio de Educación-, y Rocío Vargas y María Eugenia Barquero -secretaria y 
asistente de secretaría, respectivamente, quienes constituían el personal de apoyo logístico.

Como ilustración del fervor con que se asume el trabajo de estas primeras etapas del INIE, se destaca la disposición y solidaridad de Rocío y María Eugenia, según lo expresado por el propio Dr. Esquivel:

María Eugenia empezó a trabajar, ad honorem, en junio de 1980 hasta febrero de 1981, cuando fue nombrada como oficinista a tiempo completo. Durante 22 años sirvió a la Universidad desde el Instituto. Fue la persona confiable, honrada, servicial, siempre con una respuesta para los requerimientos de los investigadores, de otro personal o de la Dirección. Durante los primeros años del Instituto cumplió las más diversas funciones, siempre con un deseo de hacerlo de la mejor forma posible, preguntando y aprendiendo para lograr la excelencia en sus deberes. Muchas veces trabajó innumerables horas extra, que no se le pagaban $o$ se pagaban parcialmente, sin que ante nuevos requerimientos de tareas se quejara de esa falta de pago. Como quedó dicho, empezó ad honorem y, de igual manera, terminó, pues, después de pensionarse, en febrero del 2002, trabajó durante seis meses en igual condición. Esta fue una muestra más de su espíritu de servicio y del cariño que le ha mostrado a la Institución.

En 1980, llegó Rocío al IIMEC. Sirvió como secretaria ejecutiva de la Dirección hasta el 84, fecha en que ganó un concurso para el puesto de asistente de investigación. Desde esa posición y, más tarde, como analista de proyectos, fue la responsable de la organización de la sección de servicios técnicos del Instituto, que dirigió con éxito hasta que se pensionó, en el 99. Hay varias facetas de la personalidad de Rocío que es fundamental destacar. En primer lugar, su absoluto apego a los 
principios éticos y morales en el desempeño de su trabajo, que predicó con su ejemplo y que inculcó en sus subalternos. En segundo término, sus intereses gremiales verdaderos y un pensamiento social sin dobleces ni concesiones. Su faceta más importante ha sido su continuo interés por el prójimo y una preocupación sincera por el bienestar de sus compañeros de trabajo, lo que se tradujo en una acción comunal permanente.

El Instituto inicia una ruta en la que la escasez de recursos no es excusa para el estancamiento. Todo lo contrario, es un reto de crecimiento, de invención y de trabajo en equipo.

La primera tarea del Instituto consiste en realizar diagnósticos del sistema educativo costarricense, los cuales condujeron a la realización de las pruebas nacionales y las de bachillerato, en esa época a cargo del Instituto. El paradigma cuantitativo marca sus primeros pasos, dados con rigor y precisión científica. Entre otros estudios, se aborda la educación nacional en sus diversos niveles; se valoran los aprendizajes en las asignaturas básicas; se validan instrumentos; y se estudian las condiciones del profesorado, sus metodologías y técnicas.

Una segunda etapa podría señalarse como aquella en la que, al tomar conciencia de la complejidad de los problemas educativos y con nuevas perspectivas teóricas, los equipos de investigación abordan el objeto de estudio con técnicas cualitativas. Es el momento de los estudios etnográficos, de minuciosos procesos de observación en las aulas, y de producción conjunta con la participación activa de las personas sujetos de investigación. Es el momento de recuperar el sistema a partir de la experiencia de los involucrados en él y a través de sus propios significados. 
En esta etapa, bajo la Dirección del Dr. Esquivel y seguido por la Dra. Campos, perspectivas cuantitativas y cualitativas conviven en el Instituto, se complementan y se enriquecen. La pluralidad crece y el abanico va adquiriendo tonalidades variadas y dialógicas.

Posteriormente y hasta el momento, a tono con el desarrollo científico y tecnológico, se analizan nuevas tecnologías de enseñanza y se diseñan materiales educativos y mecanismos para el perfeccionamiento docente y administrativo; se impulsan metodologías innovadoras, se incorpora la perspectiva de género, y se valora la dimensión política e ideológica de la educación en todos sus niveles.

Como se ve, la historia del INIE es una historia de mejoramiento, una historia de cambio, una historia de tolerancia y de pluralidad, una historia de reflexión y de replanteamiento, una historia de compromiso con el desarrollo de la educación nacional.

Desde sus inicios y hasta el 2004, el Instituto se conoce por el nombre de Instituto de Investigación para el Mejoramiento de la Educación Costarricense, con las siglas IIMEC. Debido a recomendaciones emanadas de la evaluación de la Vicerrectoría de Investigación y al hecho de que en el 99 se rompe el convenio con el Ministerio de Educación Pública, en el año 2004 pasa a denominarse Instituto de Investigación en Educación y, por ende, INIE. Se considera que esta nueva denominación resulta más acorde con las proyecciones de regionalización del Instituto y con la gama de modalidades educativas que actualmente investiga, a saber, la educación formal, la no formal y la informal.

La misión de esta Unidad Académica es realizar, con profesionalismo y ética, investigación interdisciplinaria en educación, Volumen 5, Número Extraordinario, Año 20055 
para contribuir con soluciones transformadoras, creativas e innovadoras, sustentadas en la generación de conocimiento y desarrolladas mediante una comunidad de investigadores de distintas unidades académicas de la Universidad de Costa Rica y de otras organizaciones. En sus objetivos, el INIE muestra una real preocupación por aportar respuestas a problemas y prácticas educativas, así como por contribuir con el desarrollo teórico.

Lo anterior, describe al INIE como una unidad académica interdisciplinaria, dedicada a la investigación y al estudio de la teoría y la práctica educativa, cuyas metas son el mejoramiento del sistema educativo, y la cooperación e investigación en el ámbito nacional e internacional.

En la actualidad, el INIE cuenta con 22 áreas de Investigación, conformadas por 13 programas y líneas de investigación en los que se desarrollan 31 proyectos. Brinda 15 servicios de capacitación y asesoría. Además, sus publicaciones abarcan más de 120 Informes Finales de Investigación; discos compactos educativos; la Revista Electrónica Actualidades Investigativas en Educación; libros producto de la investigación, y el documento Bibliografía retrospectiva, el cual presenta información descriptiva de los proyectos de investigación desarrollados desde 1981 y hasta el 2004.

Todo este quehacer y producción están marcados por los ejes transversales: derechos humanos, desarrollo humano, calidad de vida, tecnologías educativas, calidad de la educación, educación en valores, educación ambiental o ecología, justicia social, educación continua y diversidad. 
Si el mérito de una institución se mide por sus logros, al cumplir los 25 años, este Instituto evidencia una amplia producción. Sus valiosos aportes reflejan el espíritu y la acción de un personal comprometido y equipos de trabajo organizados. La cuantificación y proyección de estos frutos es motivo de orgullo para el Instituto y la Universidad de Costa Rica, e inspiración para el camino pendiente.

En las siguientes palabras me complace presentarles un resumen de las semblanzas del primer Director del INIE, antes IIMEC, y de las Directoras que lo sucedieron.

La cabeza de una institución es fundamental en la trayectoria de ésta, en los ambientes de trabajo que se generan y en los proyectos que se impulsan. Siendo la autoridad formal, de la cual se espera el ejercicio de un liderazgo en las diversas labores que desempeña, es responsable de la eficacia y el progreso de la Institución. Es un puente entre ésta y las diferentes instancias nacionales e internacionales. Cada director o directora imprime a la institución su propio estilo en el ejercicio de su compleja y multifacética labor, por lo que una mirada a su trayectoria es también un repaso al caminar del Instituto.

Leo, del Dr. Juan Manuel Esquivel un resumen de la semblanza escrita por Teresita Peralta Monge:

Don Juan Manuel asume funciones como primer Director del IIMEC a partir del primero de febrero de 1980 hasta el 89.

Aunque resultaría muy interesante analizar su trayectoria en la docencia o la riqueza de su producción académica y el impacto que han tenido sus publicaciones, como hoy nos ocupa la celebración del veinticinco aniversario del IIMEC, al que don Juan Manuel dedicó cerca de diez años de su vida profesional, dirigimos esta semblanza al análisis 
de su gestión como primer Director de este Instituto y al aporte dado para su consolidación.

Como uno de los hechos más significativos en el desarrollo que ha tenido el Instituto a lo largo de sus veinticinco años al servicio del sistema educativo costarricense, destacamos hoy, con profundo sentimiento de reconocimiento y agradecimiento, la labor del doctor Juan Manuel Esquivel Alfaro, su primer Director y figura fundamental en la consolidación y en el prestigio alcanzado.

Como Director del IIMEC dirigió los primeros pasos a la ejecución de proyectos que permitieran conocer, de parte de los mismos educadores costarricenses, las necesidades de investigación y los problemas educativos trascendentales de la década de los años ochenta y que aportaran, además, elementos teóricos y prácticos que orientaran el desarrollo de los procesos investigativos que realizaría el IIMEC. Es así como en el año 1981, a pesar de las limitaciones presupuestarias y de equipo se llevan a cabo los proyectos "Diagnóstico de las necesidades de investigación en la enseñanza de Español, Inglés, Francés, Matemática, Estudios Sociales y Educación Física señaladas por los docentes que trabajan en las instituciones oficiales del país" y "Diagnóstico evaluativo de la Educación Costarricense", investigaciones que hicieron que el Instituto se ganara, muy tempranamente, un espacio en la vida de la educación costarricense.

En 1989, diez años después de su fundación, al terminar don Juan Manuel su gestión como Director, el IIMEC estaba consolidado como uno de los institutos universitarios de investigación con mayor número de proyectos activos y con el mejor récord de proyectos terminados En ese momento existían cuatro programas activos: Medición Educativa, 
Educación Superior, Epistemología y Gerontología. Además se habían establecido las líneas de investigación cualitativa e investigación en el campo de la planificación curricular. En cada uno de los programas se desarrollaban varios proyectos de investigación, en los que se daban diversas tendencias en cuanto a su fundamentación teórico metodológica.

Los que tuvimos la dicha de incorporarnos al IIMEC en sus inicios, cuando recordamos nuestros primeros años en el incursionar en el campo de la investigación educativa, evocamos la figura de este primer Director como un gestor de la investigación educativa que promovía el trabajo en equipo, así como la definición conjunta de las metas de desarrollo y los medios y estrategias para su alcance; apoyaba la iniciativa y valoraba el trabajo del personal; compartía su formación y experiencia en investigación; se preocupaba por el desarrollo académico de los funcionarios a su cargo y facilitaba acciones para su concreción; con su ejemplo incentivaba a los otros a dar lo mejor de sí mismos. Estas características personales han hecho de don Juan Manuel un verdadero maestro en el más amplio sentido de la palabra y permitieron que su liderazgo como director generara un ambiente de confianza, de respeto profesional, de compromiso con el trabajo y de identificación con el Instituto.

En las diferentes etapas profesionales encontramos personas que, por su ejemplo, sabiduría y enseñanzas dejan huella en nuestras vidas. Este sentimiento hacia el maestro que ejerce el liderazgo académico e incursiona en el crecimiento profesional de su personal fue evocado recientemente por Natalia Campos, María Eugenia Barquero, Damaris Fajardo, Marta Rojas y Teresita Peralta, todas funcionarias del Instituto 
durante sus primeros años, en una reunión a la que fueron convocadas con el propósito de conocer vivencias de los inicios.

Analizar la gestión de don Juan Manuel como Director del IIMEC, solamente desde la perspectiva de lo sucedido a lo interno del Instituto en cuanto al desarrollo que él promovió, deja de lado una faceta muy importante de este período de su vida. Su preocupación por el desarrollo de la investigación educativa en nuestro país y el liderazgo que ejerció en el ámbito nacional por la búsqueda de la unión de esfuerzos para un trabajo cooperativo entre instituciones de educación superior y el Ministerio de Educación Pública. Es así como promueve la fundación de la Comisión Nacional Coordinadora de la Investigación Educativa, la cual aún hoy, después de más de veinte años, continúa dando importantes aportes para el desarrollo de la investigación educativa en nuestro país.

Además, lleva al Instituto a realizar proyectos conjuntos con otras instituciones, tal es el caso del Diagnóstico evaluativo de la enseñanza de la Matemática, con la Universidad Estatal a Distancia y el Plan piloto para la enseñanza de las Ciencias y la Matemática, con la Universidad Nacional, el Ministerio de Educación Pública y el CONICIT.

Como Director del IIMEC, se distinguió además por el apoyo que dio a organismos como el Ministerio de Educación Pública, en las áreas de investigación educativa, desarrollo curricular y evaluación de los aprendizajes, en respuesta a un compromiso como persona interesada en el mejoramiento de la educación, el que también plasmó en sus valiosos aportes cuando participó como miembro del Consejo Superior de Educación, en representación de la Universidad de Costa Rica.

Considerado como uno de los principales especialistas latinoamericanos en el campo de la medición educativa, la preocupación 
por el mejoramiento académico de los profesionales de la educación, mejoramiento que él siempre ha valorado como un factor decisivo para el crecimiento en educación de nuestros pueblos y que ha fundamentado en los procesos de investigación educativa, sigue siendo su norte.

Gracias al maestro, al educador, al investigador, al director, al amigo, por sus enseñanzas, por su ejemplo de lucha, de lealtad, de honestidad y por su visión propositiva de la misión del INIE en el contexto del desarrollo de la educación costarricense.

En las líneas siguientes resumo la semblanza de Natalia Campos Saborío, escrita por Marta Rojas Porras:

La Dra. Natalia Campos Saborío ejerce el cargo de Directora de 1989 a 1993.

Su vínculo con el Instituto se establece desde 1980, pues, en el marco del Convenio Universidad de Costa Rica - Ministerio de Educación Pública, participa como investigadora en evaluación curricular y, desde 1983, con el apoyo en tiempos asignados por la Escuela de Formación Docente, se integra al IIMEC.

La labor investigativa de Natalia está impregnada de la madurez del trabajo en equipo, solidario y reflexivo, y de la necesidad de dar respuestas prácticas a las limitaciones y carencias del sistema educativo que en los estudios se muestran. Siendo así, de esas investigaciones derivaron múltiples cursos de extensión docente y de divulgación como labor de acción social. Además la autoría y coautoría de una gran variedad de artículos publicados en revistas especializadas, nacionales y extranjeras. Complementa este aporte colectivo la producción de 6 libros.

Gran parte de la producción escrita señalada anteriormente, la lleva a cabo mientras ejerce el cargo de Directora, pues Natalia se 
propuso que las labores administrativas no la privaran de la aventura de la investigación, lo cual muestra a una mujer altamente comprometida, constante y esforzada.

En su gestión como Directora del Instituto, incentiva un ambiente de trabajo signado por el apoyo a los diversos enfoques, por el respeto a la labor de cada funcionario o funcionaria y por la posibilidad de una comunicación abierta. Con grandes dotes para la negociación y la escucha, pone todo su empeño en mantener unida una institución que crece y modifica sus modos de interacción.

Abre el panorama investigativo del INIE poniendo un énfasis muy especial en la capacitación a partir del dato derivado de los análisis o bien utilizando estos espacios como campos de recolección de información, tal es el caso del plan piloto para el mejoramiento de la enseñanza de las Ciencias y la Matemática y el trabajo con maestros y maestras de escuelas urbanomarginales.

La temática que abarca el Instituto se diversifica a áreas que atienden desde estudios con niños de alto logro en matemática, evaluación de metodologías y programas para la enseñanza del francés y del inglés, hasta aquellas menos consideradas en la investigación educativa, tales como la educación nutricional popular o el joven y el anciano en comunicación de vida.

Siguiendo la tradición, en el VI Encuentro Nacional de Investigadores en Educación, el Instituto mantiene una muy activa presencia. También, en el período de su Dirección se organiza y lleva a cabo el Seminario de Investigación Cualitativa en la Educación Latinoamericana. 
Natalia fortalece las relaciones del IIMEC con otras instancias de la Universidad, de lo cual, como ejemplo, cito el asesoramiento que se brinda a la Facultad de Odontología para la especialidad en Ortodoncia, y la colaboración con las escuelas de Nutrición y Arquitectura.

De igual manera, Natalia trabaja arduamente por mantener el vínculo con el Ministerio de Educación Pública y con otras instituciones relacionadas con el quehacer educativo del país (UNESCO, UNED, CENAL, CENADI, UNA, CONARE, SIMED, CONICIT, etc.). En el ámbito nacional participa en la formación de educadores tanto en los niveles de grado como de posgrado. Discute en mesas redondas y periódicos nacionales, asuntos pertinentes a nuestro acontecer educativo del momento.

En su paso por la Dirección del Instituto, lo proyecta, y por ende a la Universidad de Costa Rica, no sólo en el territorio nacional sino también internacionalmente con universidades y organismos de Estados Unidos, Canadá, Inglaterra, México, Panamá, Venezuela y Chile, entre otros.

De Natalia me interesa rescatar su calidad como persona en tanto colega y amiga, y su actividad como académica que se desarrolla en los tres quehaceres fundamentales de nuestra Universidad. Desde luego, Natalia la académica y Natalia la persona no son entes separados, antes bien, veo en Natalia un ser humano que ha sabido lograr una existencia integral, en la que las teorías son llevadas a las prácticas investigativas, a las prácticas de aula y a toda experiencia cotidiana, privada o pública, de una manera congruente con sus propias concepciones, valores y creencias. 
Natalia, segunda Directora del INIE ha sido una mujer plena, alegre, entusiasta, optimista... Una mujer agradecida con lo que Dios le ha otorgado y le ha permitido vivir... Una mujer dispuesta a vencer cualquier obstáculo, pero siempre apegada al camino correcto, según sus propias convicciones... Congruente, inclaudicable y luchadora... Ejemplo y motivación... Líder innata... Una huella que el INIE debe conservar, pues sus pasos por estos caminos marcaron una senda persistente en la esperanza.

La tercera Directora del INIE fue la Dra. Ileana Contreras, de quien reseño lo escrito por Rebeca Vargas:

Ha realizado investigación y ha publicado, en revistas académicas, gran cantidad de artículos relacionados con la investigación educativa y la educación matemática.

Ha asistido a numerosos cursos de especialización y ha participado como conferencista invitada y como ponente en diversos eventos académicos relacionados con su área de trabajo.

De 1994 a 1998, la Dra. Ileana Contreras Montes de Oca ocupa el cargo de Directora del Instituto. Su labor se encamina a impulsar, apoyar y fortalecer la investigación, tanto desde enfoques cuantitativos como cualitativos; así como a reforzar vínculos con la Facultad de Educación. Para ello, impulsa proyectos como el de Informática en la Educación Superior, en el que se programan actividades dirigidas a la Facultad.

También, estrecha lazos con los Programas de Posgrado en Educación y particularmente con el Programa de Doctorado en Educación, el cual estuvo adscrito al Instituto. Colabora, además, como docente en estos programas. 
La doctora Contreras representa a la Universidad de Costa Rica en la Red Universitaria de Evaluación de la Calidad (RUECA), donde se conocieron temas relacionados con la acreditación de carreras universitarias y del Programa ALFA, el cual está conformado por redes de universidades europeas y latinoamericanas que buscan la movilidad estudiantil y la gestión en investigación.

De igual manera, fortalece y amplía los lazos del INIE con instituciones nacionales y extranjeras, coordinando la Comisión de Elaboración de Libros de Texto, específicamente los módulos de Matemáticas, Ciencias y Español.

Como representante de la Universidad ante la Comisión Nacional de Investigación Educativa (CONACIE), organiza y realiza el VII Congreso Nacional de Investigadores en Educación, en 1997.

Participa como coordinadora de la Comisión que organiza el Encuentro Nacional "La Investigación Cualitativa-Etnográfica en Costa Rica: Hacia el Encuentro con un Lenguaje Común" y el V Simposio Interamericano: "La Investigación Etnográfica en el Marco de la Investigación Cualitativa en Educación", el cual representa un gran aporte a la comunidad nacional e internacional.

De lleana vale la pena que el INIE conserve su gran capacidad de análisis y las dotes de maestra que adquieren las observaciones que realiza al trabajo investigativo. Ella conjuga, en el quehacer académico crítico, una visión coherente, sistemática y actualizada, la cual es pertinente aprender. Una Directora-investigadora quien, con su fina y alta percepción reflexiva, descubre lo que para otros no es evidente, tarea y condición fundamental en las prácticas del Instituto 
La cuarta Directora del INIE fue la doctora ALICIA GURDIÁN FERNÁNDEZ, de quien reseño lo escrito por la profesora Kemly Jiménez:

Ligada al INIE desde sus inicios, la Dra. Alicia Gurdián Fernández estuvo al frente del Instituto de 1998 al 2001.

Como ejemplo de su particular aporte como Directora del INIE, se destaca lo siguiente:

Gestiona y coordina el Programa de Nuevas Tecnologías de la Información y la Comunicación, abriendo el espacio para su posterior consolidación. Inicia también el Laboratorio de Computación.

Crea y coordina la Red Pro-educación: Red de Comunicación Computacional Interactiva con Producción de Software Educativo, cuyo primer producto fue "La Célula. Microcosmos de Vida", un importante aporte para la educación secundaria costarricense. Además gana el Premio "Aportes a la creatividad y excelencia", de la Florida Ice \& Farm Co., con el Proyecto "Kalydria y el Espejo de los Mil Rostros", un portal interactivo de educación ambiental, con una serie de juegos en diferentes escenarios, que trata de estimular cambios actitudinales en los niños y niñas - y también en las personas adultas- para crear conciencia del peligro y la responsabilidad que cabe a los habitantes de este planeta en su salvación y la de la humanidad entera.

Logra una gran apertura de la investigación interdisciplinaria, estimulando a los investigadores de diferentes unidades académicas de la Universidad a inscribir sus proyectos de investigación en el Instituto. Abre también nuevos temas de investigación, como el ambiental.

Promueve y logra la coordinación de otros proyectos interdisciplinarios con: Salud Pública, Derecho, Cómputo, Matemáticas y Geografía. 
Fortalece y amplía los lazos académicos del IIMEC con instituciones nacionales y extranjeras, como la Universidad Nacional Autónoma de México, (UNAM), el Instituto Latinoamericano de las Naciones Unidas contra el Delito (ILANUD), la UNICEF, el CRESALC y el PREAL.

Compila el libro "Una Mirada Crítica de la Educación", con el que se crea la colección "Yigüirro" para publicaciones del Instituto, con la Editorial de la Universidad de Costa Rica genera proyectos de colaboración horizontal con México, Argentina y Chile.

Fortalece y coordina el Programa de Investigación en Educación Superior (PIES).

Crea el Programa de Investigación en Educación y Género.

Promueve el Proyecto de Talleres dirigidos a profesores de Ciencias y Biología de Segunda Enseñanza, como proyecto de Extensión Docente, en el marco del Convenio entre la Universidad de Costa Rica- Ministerio de Educación- Centro Nacional de Didáctica. Éste contemplaba 21 talleres en diferentes regiones del país.

Como se ve, durante su gestión como Directora del hoy INIE, la Dra. Gurdián incentiva proyectos de investigación en áreas nuevas, da un importante impulso a la investigación cualitativa, al desarrollo de las nuevas tecnologías de la información y la comunicación, así como a la apertura de nuevos espacios de relación con instituciones nacionales y extranjeras.

De espíritu lúdico y travieso, fuerte y de gran entereza, con vehemencia defiende sus principios, es dialógica, creativa y osada. De igual manera que se sorprende ante las maravillas de la naturaleza o se emocionada por un partido de fútbol, un baile, una canción, el libro que acaba de leer, se compromete con la polémica académica, la 
investigación realizada o el éxito de una estudiante. Por tanto, su vida universitaria ha sido altamente productiva, tanto en los puestos de autoridad ejercidos, como en su labor docente y en la gran variedad de temáticas abordadas en sus investigaciones y publicaciones.

Así es Alicia, multifacética y disciplinada, atrevida y tierna, responsable y sincera, creativa y tenaz, investigadora siempre y generosa académicamente. Es de las personas que dejan huellas profundas en lo que hacen y de las que podemos esperar muchas sorpresas más. Porque como ella misma dice, aún le quedan muchos temas pendientes por investigar y reflexionar para generar más preguntas y retos que giren alrededor de la tesis central de su obra: la educación es política, es histórica y es práctica social.

En la necesidad de encontrar una respuesta clara al "para qué" de ese quehacer humano, ético, profesional, que es LA EDUCACIÓN, no hay duda de que Alicia, con su vitalidad personal y académica, continuará estimulándonos, invitándonos y retándonos a responder a tantas y tantas preguntas pendientes y nuevas.

En la actualidad, desde el 2001, la Dra. Lupita Chaves Salas ejerce como Directora del Instituto. Sobre ella resumo lo escrito por Julieta Castro:

Lupita Chaves, educadora, catedrática universitaria y constante investigadora, se caracteriza por su tenacidad y jovialidad, por su trato agradable e igualitario, por su apoyo incondicional con la gente, por el respeto y la atención a las preocupaciones ajenas, por ofrecer respuestas pertinentes y adecuadas.

De amplia trayectoria en docencia, investigación y acción social, la Dra. Chaves ha participado en trabajos investigativos relacionados con 
diversos ejes temáticos, entre los que menciono los relacionados con los procesos formativos, en las cuales se devela la acción pedagógica para trasformarla; huellas en los procesos de formación en la vida profesional de maestras de escuelas públicas; la investigación-acción para transformar los procesos educativos que emergen en la cotidianidad del centro educativo. Ha coordinado actividades de extensión docente y asesoramientos, y ha organizado gran cantidad de talleres y simposios.

Algunos ejemplos de su participación como Directora del INIE, se citan a continuación:

Gestiona una evaluación interna y externa de la labor del Instituto con el propósito de redefinir sus objetivos, su visión y su misión. Al iniciar sus funciones como Directora en el 2002, solicita y somete al Instituto a una evaluación interna de parte del personal administrativo y de investigación, y a otra externa realizada por la Vicerrectoría de Investigación. Los resultados que brinda el proceso evaluativo le permiten visualizar logros, fortalezas y debilidades, y hacen posible hacer un alto en el camino y mirar hacia atrás para construir el futuro y, a partir de reflexiones académicas, elaborar un plan estratégico quinquenal que respondiera de manera objetiva a las demandas y necesidades actuales y futuras de la sociedad. En la concreción de este plan involucra a autoridades universitarias, exdirectores, personal administrativo y de investigación. Como resultado se redefinen los objetivos y se postula la visión y la misión del Instituto; se concretan políticas de investigación, acción social y gestión administrativa, así como áreas estratégicas, programas y proyectos de acción mediante procesos participativos y de discusión académica. 
Promueve la creación de un nuevo Programa de Mejoramiento de los Procesos de Enseñanza y Aprendizaje de educación preescolar, primaria y educación secundaria, tanto en el nivel formal como informal.

Gestiona el Programa Educación y Cultura.

Logra la coordinación inter-institucional con otras universidades e instituciones públicas y privadas. Entre éstos destaca el nuevo convenio con el Ministerio de Educación Pública, firmado en el año 2003. Mediante el mismo se han desarrollado numerosas propuestas en la enseñanza de las matemáticas y del inglés para la educación secundaria; disciplina en el aula; investigación-acción en diversas instituciones educativas del país; una valoración sobre los 200 días lectivos y sobre políticas educativas.

Gestiona el proyecto "Red Interinstitucional para la niñez de Costa Rica, con la participación de instituciones dentro y fuera de la Universidad de Costa Rica.

Logra financiamiento externo mediante la participación en concursos ante La fundación Costa Rica-Estados Unidos de América para la Cooperación, así como con la Coordinación Educativa Cultural en Centro América.

Fortalece las relaciones con organismos extranjeros como los gobiernos de México, Japón y España, con los que se han establecido intercambios con académicos que colaboran con los deferentes programas que conforman el Instituto.

Incrementa los proyectos de investigación, donde se combinan de manera creativa y enriquecedora diversas disciplinas.

Motiva constantemente para que los investigadores participen en congresos y actividades académicas dentro y fuera del país. 
Logra el acercamiento de otras Escuelas de la Universidad, entre las que se citan, Lenguas Modernas, Matemáticas, Psicología, Comunicación Colectiva, Historia y Sociología, incluyendo las Sedes de Guanacaste, el Atlántico y, a partir del presente año, la Sede de Occidente, sin perder de vista las Escuelas que conforman la Facultad de Educación.

La Dra. Chaves, así como el resto de Directores y Directoras del Instituto, ha tenido presente la misión que esta Universidad debe cumplir, la cual debe encaminarse a propiciar el avance del conocimiento en su máxima expresión y responder, de manera efectiva y eficiente, a las necesidades que genera nuestra sociedad. Por este motivo, el trabajo del Instituto lo han fundamentado en los tres ejes que sustentan el quehacer de esta Universidad, a saber, la Investigación, la Acción Social y la Docencia, los que, entrelazados, contribuyen al desarrollo humano integral, y a la construcción de una sociedad más justa, solidaria, participativa y democrática.

Esta reseña del Instituto y de las semblanzas de las personas que han tenido o tienen a cargo su Dirección constituyen una fotografía panorámica de su acontecer y desarrollo. La evidencia de un trabajo que se construye en el accionar colectivo, en el que cada individuo ha aportado lo mejor de sí mismo para concretar una historia institucional plural y a la vez convergente. Un encuentro de voces amigas que prolonga, en un eco que se extiende hacia adelante, un camino hacia la luz, una dirección hacia la ternura y un sendero para el encuentro de la promesa de un mejor porvenir...

Muchas gracias y felicidades en esta fiesta académica de cumpleaños. 
